\title{
COMMUNITY DETECTION USING INTER CONTACT TIME AND SOCIAL CHARACTERISTICS BASED SINGLE COPY ROUTING IN DELAY TOLERANT NETWORKS
}

\author{
Nikhil Gondaliya ${ }^{1}$, Dhaval Kathiriya ${ }^{2}$ \\ ${ }^{1}$ Information Technology Department, G H Patel College of Engineering \& \\ Technology, Bakrol Road, Vallabh Vidyanagar, India \\ ${ }^{2}$ Director, Anand Agriculture University, Anand, India
}

\begin{abstract}
Delay Tolerant Networks (DTNs) where the node connectivity is opportunistic and end-to-end path between any pair of source and destination is not guaranteed most of the time. Hence the messages are transferred from source to destination via intermediate nodes on hop to hop basis using store-carry-forward paradigm. Due to quick advancement in hand held devices such as smart phone and laptop with support of wireless communication interface carried by human being, it is possible in coming days to use DTNs for message dissemination without setting up infrastructure. The routing task becomes challenging in DTNs due to intermittent network connectivity and the connection opportunity arises only when node comes in transmission range of each other. The performance of the routing protocols depend on the selection of appropriate relay node which can deliver the message to final destination in case of source and destination do not meet at all. Many social characteristics are exhibited by the human being like friendship, community, similarity and centrality which can be exploited by the routing protocol in order to take the forwarding decisions. Literature shows that by using these characteristics, the performance of DTN routing protocols have been improved in terms of delivery probability. The existing routing schemes used community detection using aggregated contact duration and contact frequency which does not change over the time period. We propose community detection through Inter Contact Time (ICT) between node pair using power law distribution where the members of community are added and removed dynamically. We also considered single copy of each message in entire network to reduce the network overhead. The proposed routing protocol named Social Based Single Copy Routing (SBSCR) selects the suitable relay node from the community members only based on the social metrics such as similarity and friendship together. ICTs show power law nature in human mobility which is used to detect the community structure at each node. A node maintains its own community and social metrics such as similarity and friendship with other nodes. Whenever node has to select the relay node then it selects from its community with higher value of social metric. The simulations are conducted using ONE simulator on the real traces of campus and conference environments. SBSCR is compared with existing schemes and results show that it outperforms in terms of delivery probability and delivery delay with comparable overhead ratio.
\end{abstract}

\section{KEYWORDS}

Delay tolerant networks; single copy routing scheme; social based routing

\section{INTRODUCTION}

The DTNs [1-3] are the evolution of Mobile Adhoc NETworks (MANETs) [4] where they are characterized by long transmission delays, frequent network partitions, intermittent connectivity 
and a lack of end-to-end connectivity between source and destination [5]. The communications rely on hop to hop basis and it employs store-carry-forward mechanism for message transmission until the message is reached to the final destination. Due to these characteristics of DTNs, the routing becomes very challenging task and its performance depends on the selection of appropriate relay node which gives guaranteed delivery of the messages. Even routing protocols of MANETs fail to work here as they need a continuous connected path between the source and the destination.

Our main focus is on specific application of DTNs known as the Pocket Switched Networks (PSNs) [6] where mobile devices such as smart phone, PDA and palmtop are carried by human being to provide the communication in campus and conference environment without need of setting infrastructure. The movement pattern of mobile nodes exhibit many social characteristics of human being such as community, friendship, similarity and centrality which remain stable than the mobility of the node. The researchers have also analyzed [7-9] the probabilistic distribution of Contact Time (CT), ICT, pause time and flight length using the movement traces of real time experiments performed in an academic scenario. ICT and CT between the node pair show power law distribution up to some extent followed by exponential decay which is exploited by several authors for message broadcasting in DTNs. As mobile nodes in DTNs are devices carried by human being also affects the performance due to their selfishness characteristic. The mobile nodes always would like to forward the message for those nodes with whom they have strong social ties.

Several routing protocols such as Epidemic [11], Spray and Wait [12], Prophet [13] and MaxProp [14] have been proposed, which make use of contact information to disseminate the messages but they assume that node connectivity is identical and independently distributed. Authors have also proposed the routing protocols such as Label [15], Simbet [16], BubbleRap [17] and Friendship routing [18] which exploit one or more social characteristics of human being as mentioned earlier in the routing decisions and proved to be efficient also called social based routing protocols. The most suitable forwarder node is selected by choosing the appropriate characteristics. For example, BubbleRap uses centrality or community to take the forwarding decision where Simbet makes use of similarity and betweenness together to select the relay node. The social characteristics such as centrality, similarity and friendship can be easily derived through well-defined process but community formation is difficult to describe.

The works in [19-20] detect a community structure using CT and ICT between node pairs. Each node maintains an aggregated CT and ICT with other nodes. If an aggregated value of CT and/or ICT are higher than specific pre-defined threshold value then the node is added in the list of members of its community otherwise not. But an aggregated value is not a correct measure to form a community structure which may lead to creation of false connections. S. Batyabal et al. [21] proposed a technique to determine the threshold value using power law nature of CT and ICT and construct a community structure from the mobility traces. Authors also utilized these information in routing decisions and proved that it outperformed than existing schemes. Each node maintains its own community locally and forwards the messages to all those nodes which belong to the same community of the message destination. This routing protocol does not use any metric to select the most appropriate relay node from the list of members of the community.

Majority of the above routing protocols have used multi copy message or flooding approach to disseminate the messages in the network. Obviously they can improve the chances of delivering the messages with minimum delivery delay but incur higher overhead due to unnecessary transmission of the message. The existing work [21] lacks applying of social based metrics to select the most appropriate relay node among the members of a community and routing protocol is implemented as multi copy scheme. 
In this paper, we propose the social based routing protocol which utilize the community structure along with social metrics such as similarity and friendship to select the most appropriate relay node. The community is formed by exploiting the power law nature of ICTs at each node. We use singly copy of each message in the network and compared with existing routing protocols. The simulations are conducted using ONE simulator on real traces of campus and conference environment to show the effectiveness of SBSCR. The contributions of this paper are summarized as follows:

1) We propose construction of the community at each node using power law nature of ICTs.

2) We also propose social metric based on characteristics such as Jaccard similarity coefficient and friendship to select the most suitable forwarder amongst the members of a community.

The reminder of the paper is organized as follows. Section 2 surveyed on the related work. In Section 3, the experimental data sets and power law characteristics of ICTs are described. A community detection process is explained in Section 4. Social based metrics and the proposed routing protocol are presented in Section 5 and Section 6 respectively. Section 7 and 8 discuss experimental parameters and results and analysis respectively. Finally conclusions are drawn with future work in Section 9.

\section{RELATED WORK}

The DTNs have intermittent network connectivity and lack of end-to-end path which make the routing task most difficult. Authors have proposed many routing protocols for such type of environment which can be categorized as single copy scheme and multicopy scheme [22]. This is based on number of message copies in network at any time instance. Several authors have categorized them as naïve replication and utility based routing protocols [23]. The naïve replication method either floods the message to every encountered node or controls the flooding up to some $\mathrm{N}$ number of copies of each message. The utility based methods use one or more utilities to take the decision whether the message is forwarded or not.

Direct delivery [24] and First Contact [25] routing are the example of the single copy scheme where source node will carry the message until it is delivered to the destination. They have lowest overhead in the network but its performance in terms of delivery ratio and delay is very poor if source node can't come in the proximity of the destination. Epidemic [11] is multicopy routing protocol in which the node floods the message to all those nodes which come in direct contact. It performs the best in terms of delivery probability only if there is no restriction on buffer storage and bandwidth. Spray and Wait [12] floods limited number of copies (L) of each message. Maximum L number of nodes will get the copy of the message and wait for direct encounter of the destination node. This protocol shows lower delivery probability than Epidemic due to controlled flooding but reduces the network overhead.

Prophet [13] is example of utility based routing protocol which forwards the message using delivery predictability metric. Each node maintains the delivery predictability value with other nodes and it is updated for each encounter. If delivery predictability value of the relay node is higher then the message is forwarded otherwise not. Social based routing protocols are in the family of utility based routing category.

Social based routing has become an active area of research since last many years which is based on the theory of Social Network Analysis [26] to study the relationship among the people. One advantage of using social-based forwarding in DTNs is that social characteristics tend to be less unstable than the node mobility [27].First proposal in social based routing was Label [15] where 
the nodes are affiliated to the social community and the message is forwarded only to those nodes which belong to the community of the message destination. However, it needs a community information available in prior. Simbet [16] routing protocol uses similarity and betweenness centrality metrics together to select the forwarding node. They give equal importance to both the utilities and the message is replicated if peer node has higher value of the utility than the message carrier node. It is not always possible to predict the future encounters so, node with higher value of utility may fail to deliver the message. BubbleRap [17] protocol is based on the idea of global/local centrality and community. The global centrality and local centrality are used to know more popular node in the network as a whole and inside community respectively. Each node also maintains its own community list and centrality. When node encounters with another node and if it belongs to community of message destination then message is forwarded. Otherwise message is forwarded based on higher global centrality value and finally it is delivered to one of the members of its community or destination. This scheme may not work when node belongs to the community whose all members are with low global centrality value [21]. Friendship routing [18] construct friendship community using the social pressure metric which measures the quality of friendship based on their long lasting and regular contact.

Many of the above routing protocols utilize the community information in order to take the forwarding decisions. Moreover, the performance of social based routing protocols mainly depends on how the community structure is formed. T. Hossmann et al. [20] proposed a method to construct community structure based on aggregated contact duration between the nodes. In [17], P. Hui et al. identified an online decentralized community detection technique using contact duration between node the pair. If aggregated contact duration of the same node pair exceeds than predefined threshold value then both nodes add each other in its community list. All of these works have used contact duration and contact frequency in order to construct a community structure at each node which may not be the correct criterion as community structure evolves over the time. As community structure changes over the time which is not considered in the existing techniques of community detection. S. Batyabal et al. [21] proposed a method of community detection by exploiting the distribution of ICTs and CTs from the mobility traces. An aggregated ICTs and CTs of all node pairs follow power law distribution with exponential cut off [28-29]. Authors also utilized these knowledge in the routing decisions where each node maintains the list of members in its community. The message is forwarded to all members of a community and finally it will reach to the destination. This concept does not utilize any other social metric along with community information to find the most appropriate relay node which gives guaranteed delivery of the messages.

Our focus in this paper is to detect the community structure using only ICTs distribution between the node pairs. It can be assumed that there is plenty of bandwidth with nodes so, the distribution of CTs may not help here to identify community. The next Section describes power law characteristics of ICTs in the data sets of real time experiments for human mobility.

\section{EXPERIMENTAL DATA SETS AND ANALYSIS FOR ICT}

To analyze the behavior of SBSCR, we have used experimental data sets from an academic scenario which can be categorized into two distinct environments: campus and conference. These data sets are widely used by majority of social based routing protocols as discussed in Section 2 and publically available on CRAWDAD portal [30]. The following part briefly describes about the data sets and Table 1 shows their characteristics.

1. Cambridge: The iMote devices were distributed to 36 students in the university campus among four groups of students: $1^{\text {st }}$ and $2^{\text {nd }}$ year UG students, $\mathrm{PhD}$ and master students. The experiment was lasted for 11 days. 
International Journal of Ad hoc, Sensor \& Ubiquitous Computing (IJASUC) Vol.7, No.1, February 2016

2. PMTR: The faculty members, $\mathrm{PhD}$ students and technical staff members were distributed dedicated device to record the data. Total 49 participants were selected and the experiment was performed for 19 days.

3. Infocom05: Participants of the conference were distributed 41 devices and experiment was conducted for 3 days. They were from different regions, academic affiliations and research groups.

4. Infocom06: It is mostly similar to Infocom05 but on larger scale with 98 participants.

Table 1. Characteristic of real trace data sets

\begin{tabular}{|c|c|c|c|c|c|c|}
\hline Dataset & $\begin{array}{c}\text { Number } \\
\text { of nodes }\end{array}$ & $\begin{array}{c}\text { Duration } \\
\text { (in days) }\end{array}$ & $\begin{array}{c}\text { Number of } \\
\text { communities }\end{array}$ & $\begin{array}{c}\text { Device } \\
\text { type }\end{array}$ & $\begin{array}{c}\text { Total no. of } \\
\text { ICTs }\end{array}$ & $\begin{array}{c}\text { Shape } \\
\text { parameter } \\
(\boldsymbol{\alpha})\end{array}$ \\
\hline Cambridge & 36 & 11 & 4 & iMote & 8772 & 1.41 \\
\hline PMTR & 49 & 19 & 3 & PMTR & 11895 & 1.43 \\
\hline Infocom05 & 41 & 4 & 11 & iMote & 19253 & 1.51 \\
\hline Infocom06 & 98 & 4 & 14 & iMote & 156322 & 1.49 \\
\hline
\end{tabular}

To avoid the unbiased decision, the data sets are chosen from two distinct environments for analysis purpose. Campus data sets where nodes are more familiar with each other and form community structure with greater number of members. In conference data sets, nodes are not known to each other and it becomes difficult to detect community. Due to this, conference data sets show large number of communities with small membership than campus data sets.

The two most important characteristics of human mobility such as ICT and CT which have great impact on the routing performance and it has been studied in [9]. Figure 1 shows complementary cumulative distribution function (ccdf) of ICTs for Cambridge and Infocom05 data sets on loglinear scale. It is observed that they follow power law distribution upto a characteristic time then it exhibits an exponential cut off[21]. Power law distribution is also called Pareto distribution (8020 law) used in many situations in which equilibrium is found in the distribution of the small to the large. For example, in case of wealth or income in any country we can say that $20 \%$ people own $80 \%$ of the wealth.

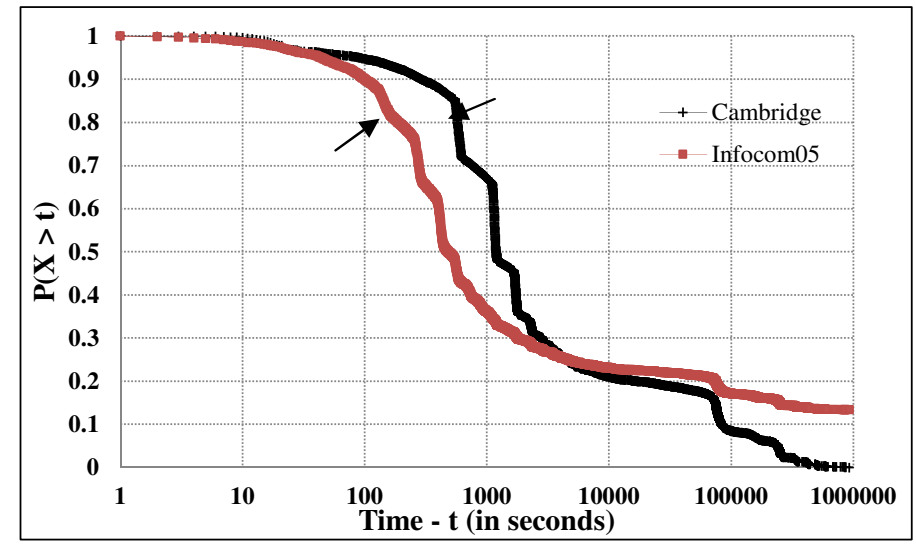

Figure 1. CCDF of ICTs for Cambridge and Infocom05 data sets

From Figure 1, it can be seen using arrow that $20 \%$ of ICTs are less than 800 seconds in Cambridge data set where they are less than 300 seconds in Infocom05 data set. Due to confined 
area in conference environment, people meet more likely to each other with smaller ICTs than campus environment. We can extract all ICTs where $\mathrm{P}(\mathrm{X}>\mathrm{t})=0.8$ according to Pareto distribution. The community detection method use this nature of ICTs to form the community structure by analytically finding appropriate threshold value of ICT at each node periodically. The similar characteristics of ICTs is also observed in remaining two data sets. The next Section describes that how node decides whether other node is added into its community or not.

\section{COMMUnity DeTECTION BASED ON ICTS}

This Section discusses on how to construct the community structure at each node locally based on ICTs of encountered nodes. The main task is to find the threshold value of ICT in unbiased way which determines that whether node can be added into community or not. Each node maintains the vector of ICTs for all the encountered nodes. For example, node $X$ maintains vector $E_{X Y}=\left\{t_{1}\right.$, $\left.t_{2}, t_{3}, \ldots, t_{n}\right\}$ for another node $Y$ at specific point of time. This ICT vector is kept in increasing order means $t_{1} \leq t_{2} \leq t_{3}, \ldots, \leq t_{n}$. Also each node maintains characteristic time ( $\mathrm{ICT}_{\mathrm{ct}}$ ) for each encountered node based on Pareto rule of 80-20. So, the characteristic time for vector $E_{X Y}$ is calculated as $\left|E_{X Y}\right| * 0.2$ (where $\left|E_{X Y}\right|$ is size of vector) which is $t_{3}$ and similarly node holds the same for all encountered nodes. The following part explains the entire process with example.
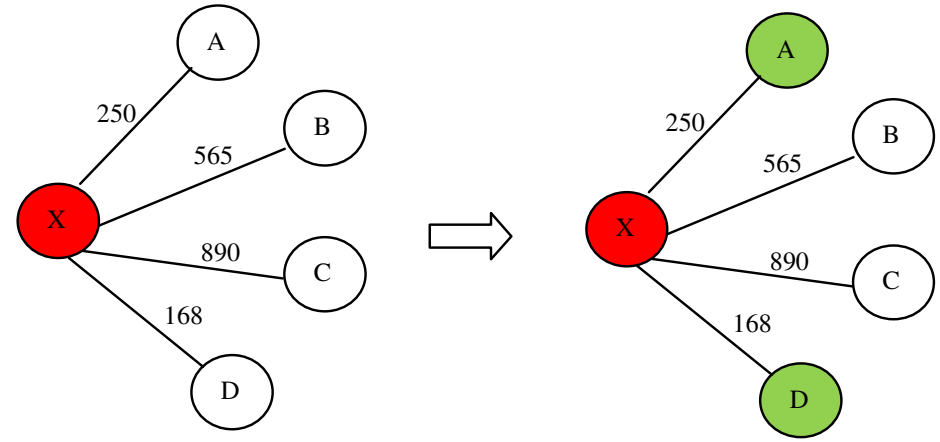

Figure 2. Community detection at node $\mathrm{X}$

Assume that node $\mathrm{X}$ is encountered with four nodes namely $\mathrm{A}, \mathrm{B}, \mathrm{C}$ and $\mathrm{D}$ and its ICT vector are $\mathrm{E}_{\mathrm{X}, \mathrm{A}}=\{100,250,1010,1260,1705,2345,3455,4532,6538,6711\}, \mathrm{E}_{\mathrm{X}, \mathrm{B}}=\{565,1234,4567\}$, $E_{X, C}=\{890,3456,5738\}$ and $E_{X, D}=\{168,349,674\}$ respectively at specific point of time. Node $\mathrm{X}$ finds an $\mathrm{ICT}_{\mathrm{ct}}$ for each vector as specified above and it is labeled on the respective edge as depicted in Figure 2.

Now we need to find an ICT threshold ( $\left.\mathrm{ICT}_{\mathrm{th}}\right)$ which is compared against $\mathrm{ICT}_{\mathrm{ct}}$ of each node pair and decision will be made to add the node in a community. As per the power law distribution of ICT, we determine the power law index and then data point $\left(\mathrm{ICT}_{\mathrm{th}}\right)$ which satisfies $P(X>t)$. The power law distribution is defined as per equation 1 as follows.

$$
p(x)=C *\left(\frac{x}{x_{\min }}\right)^{-\alpha}
$$

Where $\alpha$ and $C=\frac{\alpha-1}{x_{\min }}$ are shape parameters and normalized constant respectively. $x$ and $x_{\min }$ are data point and minimum value of data point for power law shape respectively. To estimate the shape parameter, we use following maximum likelihood function.

$$
\hat{\alpha}=1+n\left[\sum_{i=1}^{n} \log \frac{x_{i}}{x_{\min }}\right]^{-1}
$$


Where $x_{i}>x_{\min }$ and $\mathrm{n}$ is number of data points which are greater than $x_{\min }$. Node $X$ accumulates $\mathrm{ICT}_{\mathrm{ct}}$ of all the encountered nodes into new vector $\left(\mathrm{CTT}_{\mathrm{x}}=\{168,250,565,890\}\right)$ to estimate $\widehat{\alpha}$ as per equation 2 by taking $X_{\min }$ to 300 . We get the value of $\widehat{\alpha}$ to 1.41 and it is replaced in equation 3 with $\alpha$ and $p(x)$ is set to 0.2 to get the $\mathrm{ICT}_{\text {th }}$ value. Hence, the determined value of $\mathrm{ICT}_{\mathrm{ct}}$ is 250 .

$$
I C T_{t h}=\left(p(x) * x_{\text {min }}^{1-\alpha}\right)^{\frac{1}{1-\alpha}}
$$

As shown in Figure 2, node $A$ and $D$ are added into the community member list of node $X$ as their characteristic time is less than or equal to the determined $I C T_{t h}$. Similarly other nodes follow the same process to calculate the $I C T_{t h}$ for community construction. Also nodes may belong to more than one community and they can be added and removed from the community as per the updated value of $I C T_{\text {th }}$ from time to time. The routing protocol utilizes this community information along with social based utility which is discussed in the next Section.

\section{Social BaSed UtiLity}

SBSCR protocol utilizes the social based utility (SBU) to select the most appropriate relay node among the members of a community. SBU is mainly made of two different utilities: similarity and friendship which are discussed in Section 5.1 and 5.2 respectively.

\subsection{Similarity Utility}

The degree of contact between nodes has great impact in terms of information dissemination [15]. Nodes with lower degree of the separation from a given node are good forwarding candidates. We use Jaccard's similarity coefficient as per equation 4 to measure similarity of the node with another node. It is defined as division between the number of community members common to both set $X$ and $Y$ divided by all the members of both communities.

\subsection{Friendship Utility}

$$
J(X, Y)=\frac{|X \cap Y|}{|X \cup Y|}
$$

We use concept of delivery predictability [12] to determine the friendship between the node pair. Friendship $\left(F_{x y}\right)$ reflects that how likely for node $x$ to deliver a message for node $y$. When node encounters with each other then they update the friendship utility as per following equation.

$$
F_{x y}=\widetilde{F_{x y}}+\left(1-\widetilde{F_{x y}}\right) * F_{\text {init }}
$$

Where $F_{\text {init }} \in(0,1]$ is an initialization value and $\widetilde{F_{x y}}$ is the old value of $F_{x y}$. If node encounters more frequently then there will be higher value of friendship between them. The friendship value is also aged according to equation 6.

$$
F_{x y}=\widetilde{F_{x y}} * \gamma^{\text {last_ICT }}
$$

Where $\gamma \in(0,1]$ is an aging parameter and last_ICT is time elapsed since last encounter between two nodes. Each node stores ICT vector for another node and friendship value is aged based on the last ICT in the list between the same node pair when they encounter and apply equation 6 for the same. 
We combine the similarity and friendship utility by giving equal importance to both and derived following equation 7 to compute the final value of SBU.

$$
S B U_{x y}=J(x, y) * 0.5+F_{x y} * 0.5
$$

\section{DESCRIPTION OF THE PROPOSED APPROACH: SBSCR}

An algorithm 1 explains that how message is forwarded from source/intermediate node to the destination node via multiple hops. For example, node $x$ encounters with another node $y$ then node $x$ will perform the steps as depicted in algorithm 1 for all the messages in its buffer which may be intended for different destination.

Suppose message carrier node $S$ has a message to be delivered to the destination $D$. First it checks that destination of the message is member of the community of encountered nodes. Then it compares the SBU value with encountered node for message destination and if it is higher than message carrier nodethen message is forwarded otherwise not. If both the nodes, message carrier and encountered node contain the message destination in their community then message is forwarded to the node with higher SBU value. Figure 3 shows that node $S$ encounters another node $Y$ and destination $D$ is member of its community. SBU value of $Y$ is higher than $S$ so, message is forwarded to $Y$. Number besides node indicates the SBU value for message destination $D$. Afterward node $Y$ or one of its members of community has more chances to encounter the destination $D$. In this way, messages are delivered with multiple hops to the final destination.

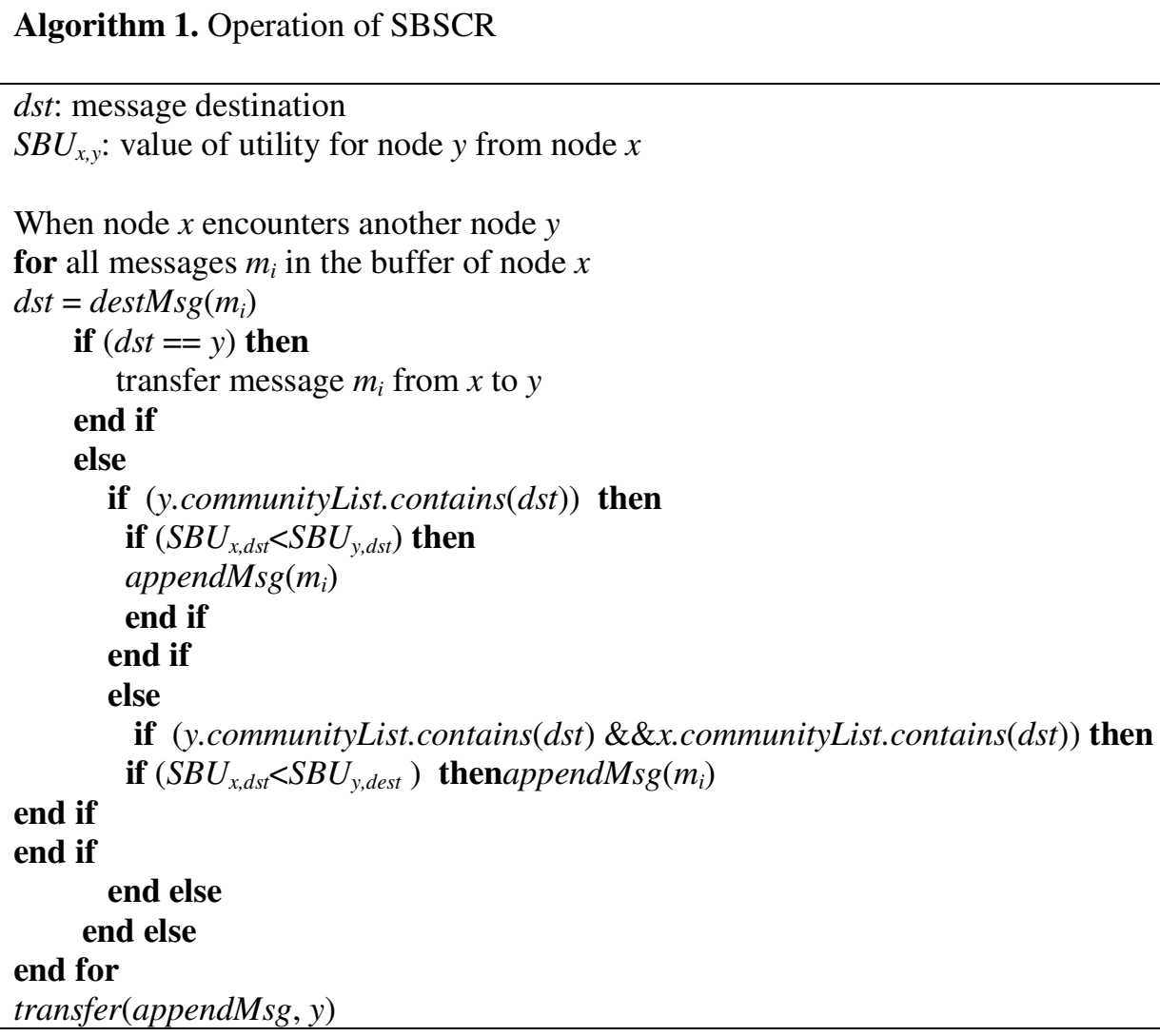




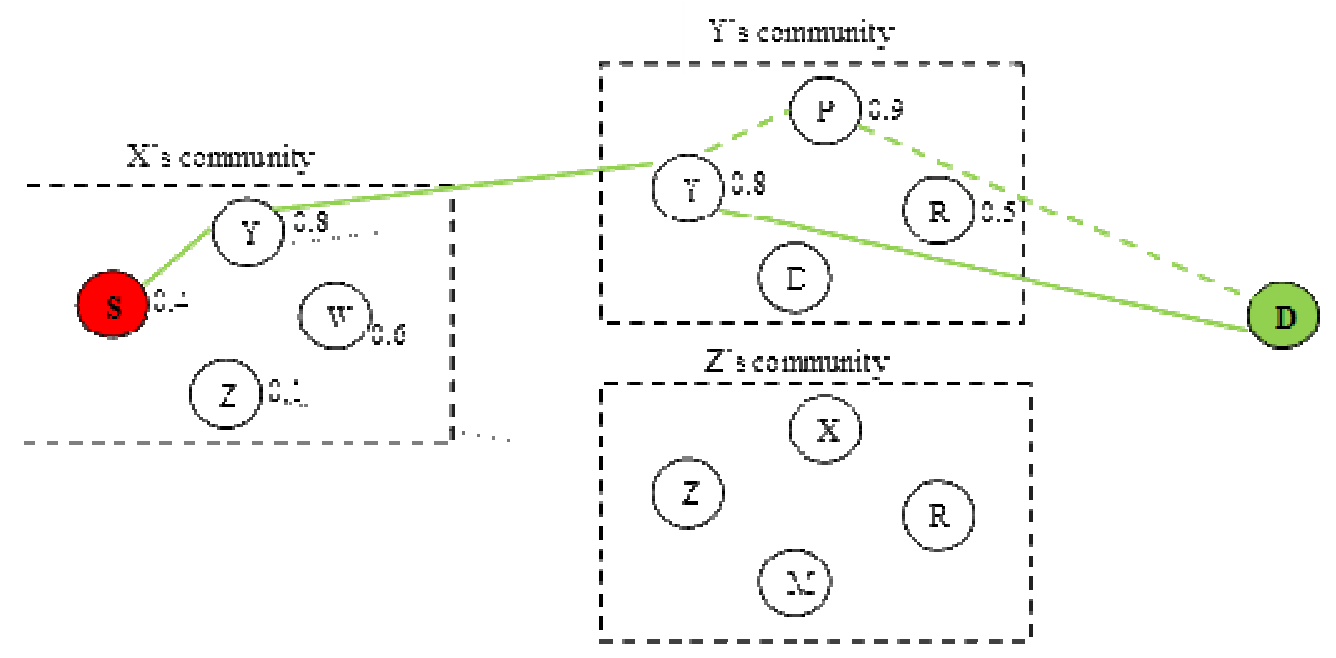

Figure 3.The transmission of a message from node $\mathrm{S}$ to node $\mathrm{D}$

\section{Experimental Setup and Performance Metrics}

The extensive simulations are performed using the ONE simulator [31] which is event driven simulator designed specifically for evaluating DTN routing protocols. It is required to set different parameters for the simulation and the routing protocols need to be compared using performance metrics. The following sub sections describe them in detail.

\subsection{Simulation Parameters}

The mobility traces are taken from real data sets as discussed in Section 3 to evaluate the performance of the routing protocols. Many parameters of the simulation need to be changed while few remain fixed. Transmission speed, message size and message generation interval are $2 \mathrm{Mbps}, 25 \mathrm{k}$ and 30-40 seconds respectively and they remain fixed if not specified explicitly.

The performance is measured using all four data sets by varying buffer size and Time-To-Live (TTL) value. The results of only one data set from each environment are shown in further Section, while the parallel conclusions can be drawn for the remaining data sets. For each value of TTL and buffer size, the simulations were run five times with different random generator seeds. SBSCR protocol is compared with three existing schemes such as Prophet, BubbleRap and Simbet. We use the configuration parameters for all the existing routing protocols as per their original implementation.

\subsection{Performance Metrics}

The following performance metrics have been used to evaluate the performance of routing protocols.

1. Delivery probability or delivery ratio: It is calculated as the ratio of the number of messages successfully delivered to the destination to that of the total number of messages generated in the network.

2. Average delivery delay or latency: Delivery delay is the time elapsed between the creations of the message at source and delivered successfully to the destination. Average delivery delay is average of delivery delay of all the delivered messages. 
3. Average overhead ratio: It is the ratio of the difference of the total number of message relayed minus delivered successfully to that of the number of messages delivered successfully. This is also a measure of the additional number of transmissions required for each message to be delivered from source to the destination.

\section{RESUlt ANALYSIS AND DiscuSSIONS}

To demonstrate the effectiveness of SBSCR, it is compared with three existing routing protocols and all of them were implemented as single copy scheme. Source node generates a single copy of each message and the same is received by the destination node. The Sections 8.1 and 8.2 discuss the result of simulations under varying buffer sizes and TTLs respectively.

\subsection{Under Varying Buffer Size}

The buffer space is limited resource in the nodes of DTN and it must be managed efficiently to improve the performance of the routing protocol. An aim of these simulations is to show the performance of protocols under varying buffer sizes. The buffer size is varied from $1 \mathrm{MB}$ to $25 \mathrm{MB}$ in interval of 2 for campus data sets and $1 \mathrm{MB}$ to $8 \mathrm{MB}$ in interval of $1 \mathrm{MB}$ for conference data sets. TTL value is set to 2 days and 1 day for campus and conference data sets respectively.
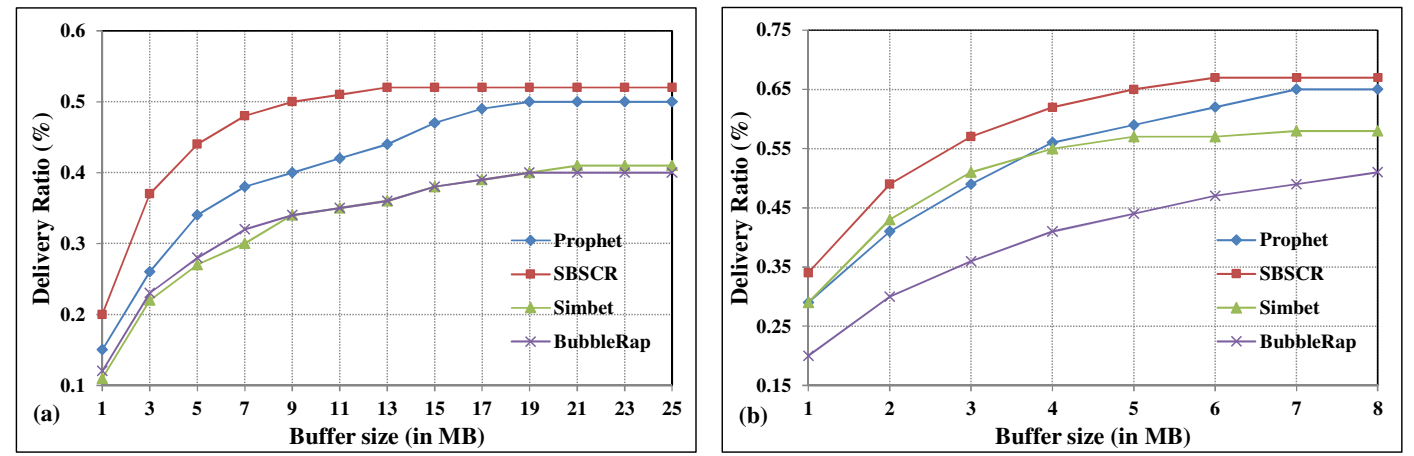

Figure 4. Average delivery ratio for (a) Cambridge and (b) Infocom05 at varying buffer size

Figure 4(a) and 4(b) confirm that delivery ratio is increasing as the buffer size increases for all the routing protocols in both the data sets. SBSCR outperforms in delivery ratio than other routing protocols and it becomes steady at buffer size of $13 \mathrm{MB}$ and $6 \mathrm{MB}$ for Cambridge and Infocom05 data set respectively. SBSCR needs the minimum buffer space to achieve the maximum delivery ratio. Delivery ratio of SBSCR is upto 45\% higher than Simbet and BubbleRap while 30\% more than Prophet in Cambridge data set. It is also higher in Infocom05 data set upto 14\% than Prophet and Simbet and $41 \%$ than BubbleRap. The performance improvement of SBSCR is due to dynamic community detection by exploiting ICT's power law distribution along with social based utility which can find the most suitablerelay node.

BubbleRap and Simbet show lower performance as they use value of ACT (Aggregated Contact Time) and the encounter information respectively to detect community structure which may not be correct criterion to add the nodes inside community [18]. Even Simbet performs better in Infocom05 data set than Cambridge data set, as more number of unique and total encounters in Infocom05 data set due to confined area and diversity of people in the experiment. 

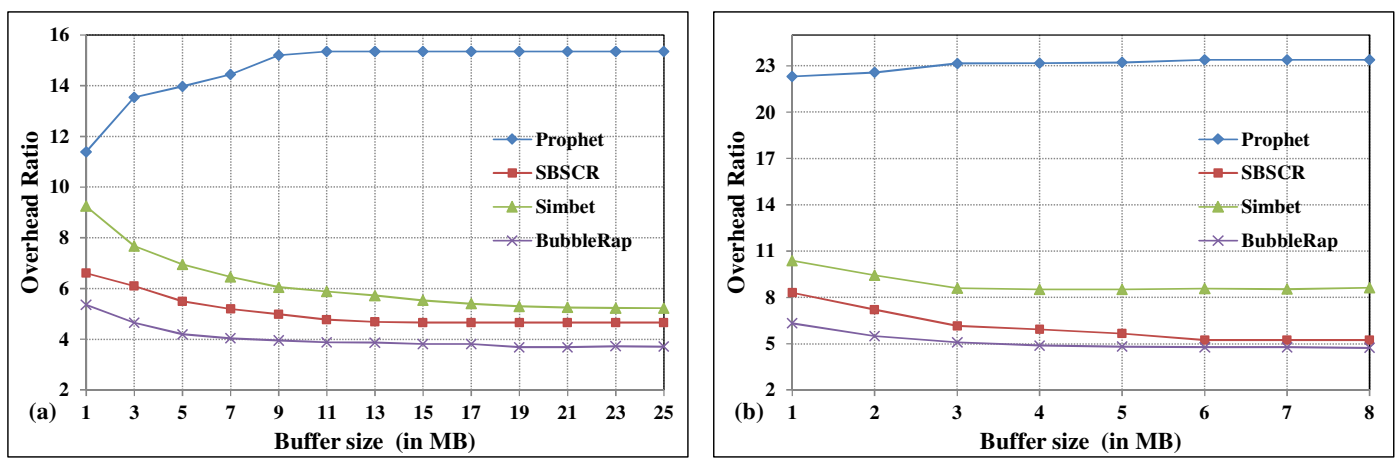

Figure 5. Average overhead ratio for (a) Cambridge and (b) Infocom05 at varying buffer size

Average overhead ratio depends on number of messages relayed and delivered successfully to the destination. When buffer size is increasing, it reduces the number of message dropping and they get more opportunity of delivering to the final destination. We have used single copy of the message throughout the network which reduces the number of transmissions than the multi copy scheme and also save the buffer space. As buffer size increases, delivery ratio is increasing and overhead ratio is decreasing which is true for all routing protocols except Prophet as depicted in Figure 5(a) and 5(b). SBSCR relays less number of messages than Prophet and Simbet but a little bit more than BubbleRap.
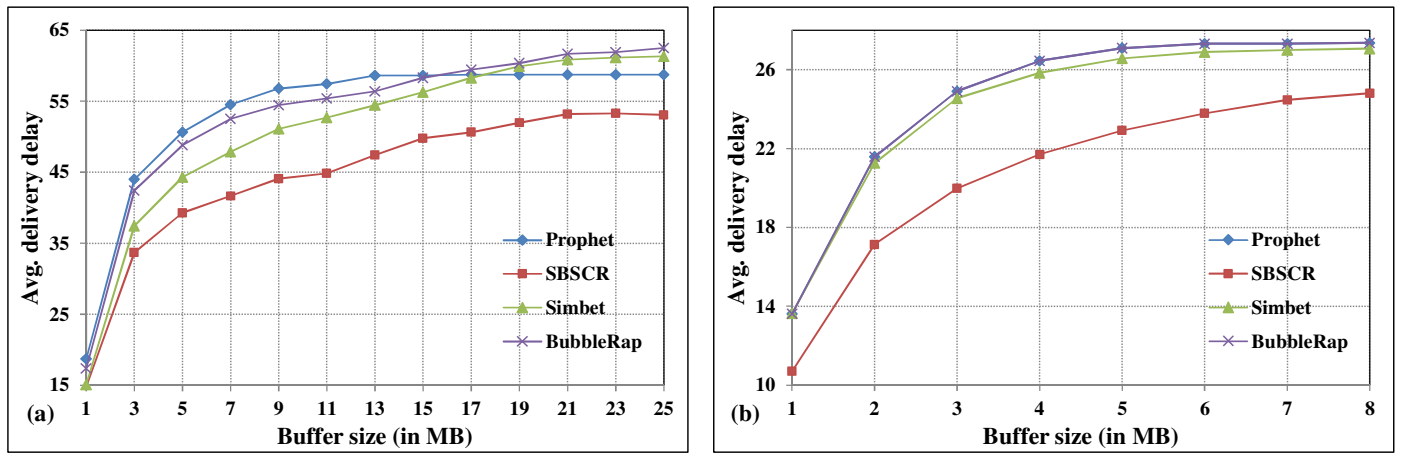

Figure 6. Average delivery delay (in Thousand Seconds) for (a) Cambridge and (b) Infocom05 at varying buffer size

Average delivery delay is affected by level of replication of the messages but all of the routing protocols used single copy of the message so, delivery delay is largely depended on the correct choice of relay node. Figure 6(a) and 6(b) demonstrate that delivery delay is increased for all the routing protocols as buffer size is increasing. SBSCR has lowest delivery delay because ICT based community detection method with social based utilities allow selection of those nodes which encounter very frequently.

\subsection{Under Varying Time-To-Live}

These set of simulations verifies that by giving more life time to the messages, they can get more opportunities to reach the destination before they get expired. TTL value is varied from 10 minutes to 1 week and 10 minutes to 2 days for Cambridge and Infocom05 data sets respectively with fixed buffer size of 10MB.

Figure 7(a) and 7(b) illustrate that SBSCR always outperforms than existing schemes at all the values of TTLs. Delivery ratio becomes stable at TTL value of 2 days and 1 day onwards for all 
International Journal of Ad hoc, Sensor \& Ubiquitous Computing (IJASUC) Vol.7, No.1, February 2016

routing protocols except SBSCR in Cambridge and Infocom05 data sets respectively. At higher value of TTL, the nodes in SBSCR can get more opportunity to select the appropriate relay node compared to others.
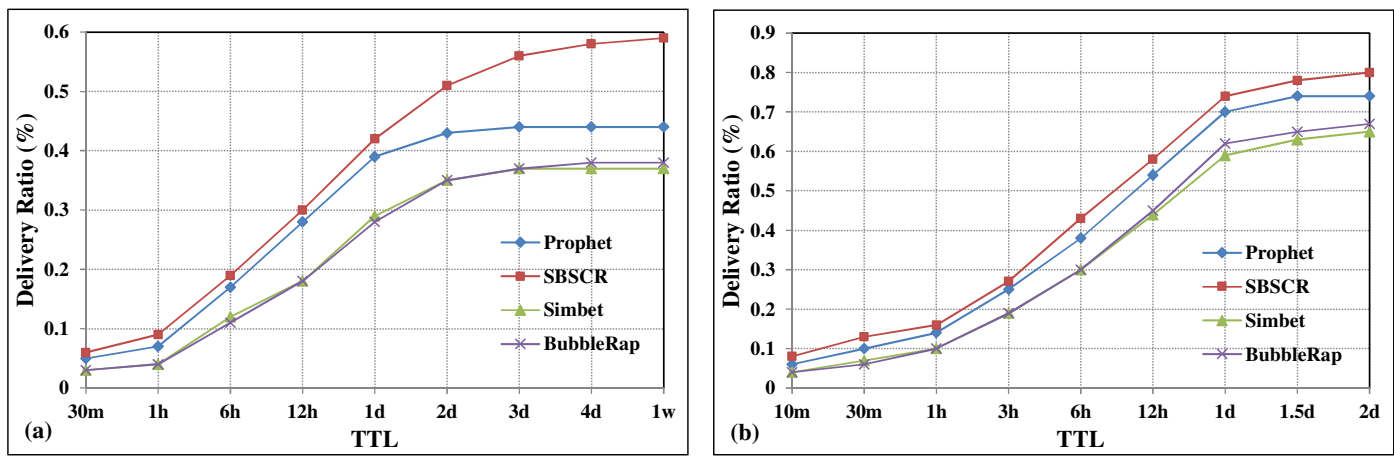

Figure 7. Average delivery ratio for (a) Cambridge and (b) Infocom05 at varying TTL
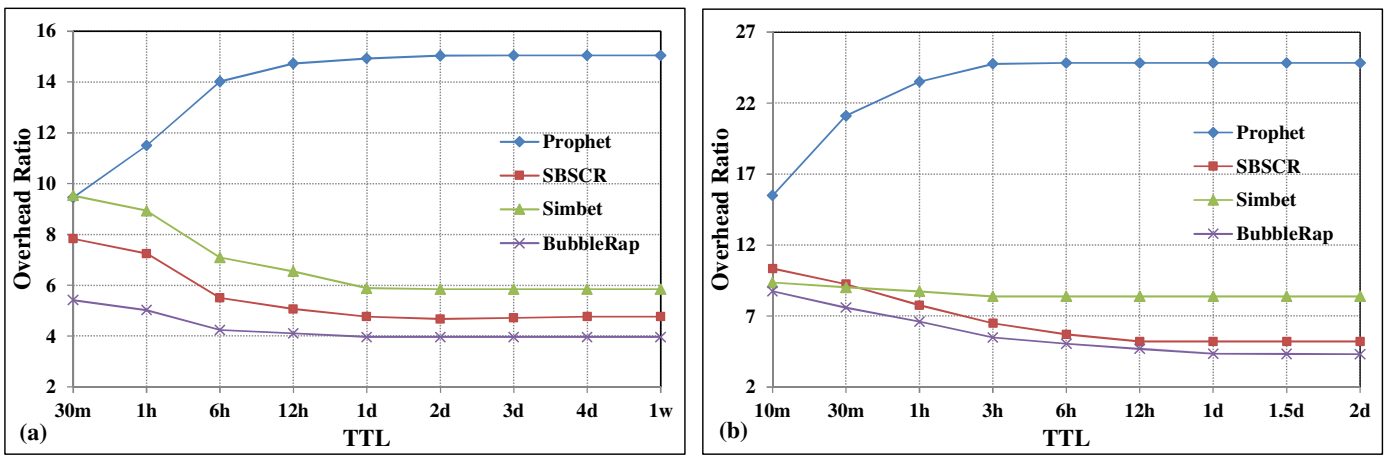

Figure 8. Average overhead ratio for (a) Cambridge and (b) Infocom05 at varying TTL

An average overhead ratio decreases for all the routing protocol except Prophet as TTL value increases as shown in Figure 8(a) and 8(b). BubbleRap has lowest overhead for Cambridge and Infocom05 data set as node forwards the message only if encountered node belongs to community of the message destination or node with higher global centrality. SBSCR shows lower overhead ratio than Simbet and Prophet routing protocols for both the data sets.
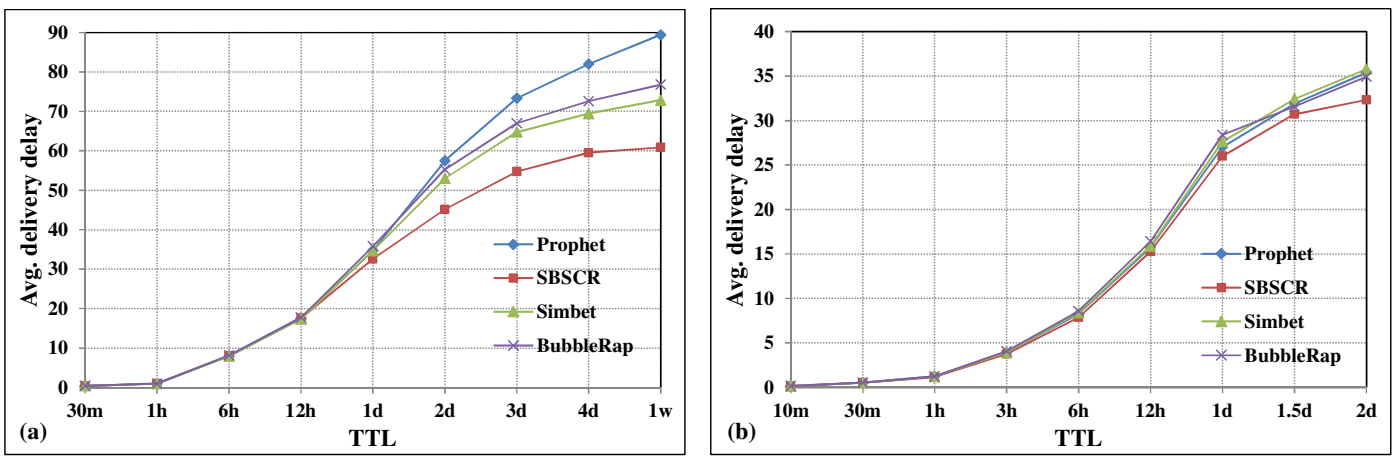

Figure 9. Average delivery delay (in Thousand Seconds) for (a) Cambridge and (b) Infocom05 at varying TTL 
An average delivery delay is very close for all routing protocols upto TTL value of 12 hours and 6 hours for Cambridge and Infocom05 data sets respectively as depicted in Figure 9(a) and 9(b). SBSCR shows lowest delivery delay than others at higher TTL values. The reason behind is that at higher value of TTL, the nodes can find more opportunities to select the most appropriate relay nodes which meet to the message destination in as fast as possible.

\section{CONCLUSIONS AND FUture WORK}

In this paper, we presented community detection approach by exploiting the power law distribution nature of ICTs between the node pairs. The ICTs threshold value is identified analytically from time to time according to Pareto rule of $80-20$ which denotes that $20 \%$ of ICTs between node pair are less than determined threshold value and node can be added into community. Social based utility is also suggested that is made of similarity and friendship value. We tested the performance of SBSCR by comparing with the existing protocols using single copy scheme on real trace data sets of campus and conference environment by varying buffer size and TTL. Our result confirmed that SBSCR outperformed in terms of delivery ratio and delivery delay than the existing routing protocols at varying buffer size and TTL in the data sets of both the environments with comparable average overhead ratio.

The proposed routing protocol SBSCR exploits one hop community information along with social metric but in future we can utilize two hop information in order to make the forwarding decisions by routing protocol. This will be helpful in knowing the most appropriate relay node which can improve the delivery probability up to some extent.

\section{REFERENCES}

[1] S Farrell S \& V Cahill, Delay and disruption tolerant networking. Artech House, Inc. (2006)

[2] IRTF: RFC 4838 - DTN Architecture, 2007. Available at http://www. ietf.org/rfc/rfc4838.txt

[3] IRTF: Delay Tolerant Networking Research Group, 2009. Available at http://www.dtnrg.org

[4] C Perkins, Ad hoc networking, Addison-Wesley, (2001)

[5] E Daly, M Haahr, The challenges of disconnected delay-tolerant MANETs, Ad Hoc Netw., Elsevier, pp. 241-250, (2010)

[6] A. Chaintreau, P. Hui, J. Crowcroft, C. Diot, R. Gass, and J. Scott. Pocket switched networks: Realworld mobility and its consequences for opportunistic forwarding. Technical Report UCAM-CL-TR617, University of Cambridge, Computer Laboratory, February (2005)

[7] I Rhee, M Shin, S Hong, K Lee,SJ Kim, S Chong, On the levy-walk nature of human mobility. IEEE/ACM Transactions on Networking, 19(3), 630-643 (2011)

[8] MC Gonzalez,CAHidalgo,A Barabasi, Understanding individual human mobility patterns. Nature, 53(7196), 779-782 (2008)

[9] T Karagiannis, J Boudec, \& M Vojnovi, Power law and exponential decay of inter contact times between mobile devices. In Proceedings of the ACM MobiCom, pp. 183-194, (2007)

[10] A Chaintreau, P Hui, C Diot, R Gass, J Scott \& J Crowcroft, Impact of human mobility on opportunistic forwarding algorithms. IEEE Transactions on Mobile Computing, 6(6), pp. 606-620 (2007) 
International Journal of Ad hoc, Sensor \& Ubiquitous Computing (IJASUC) Vol.7, No.1, February 2016

[11] A Vahdat, D Becker, Epidemic routing for partially connected Ad Hoc networks. Technical report CS2000-06, Department of Computer Science, Duke University, Durham, NC.(2000)

[12] T. Spyropoulos, Psounis, Konstantinos, C. S. Raghavendra,Efficient routing in intermittently connected mobile networks: The multiple-copy case. IEEE/ACM Transactions on Networking, 16(1), 77-90 (2008)

[13] A Lindgren,A. Doria and O.Schelen, Probabilistic routing in intermittently connected networks. InProceedings of ACM SIGMOBILE Mobile Computing and Communications Review, 7(3)), pp. 19$20(2003)$

[14] J Burgess, B Gallagher, D Jensen, BN Levine, Maxprop: Routing for vehicle-based disruption-tolerant networks. In Proceedings of the IEEE INFOCOM, vol. 2,pp. 1688-1698 (2006)

[15] P Hui, J Crowcroft, How small labels create big improvements. IEEE pervasive computing and communications workshops (PerCom’07) (pp. 65-70), 19-23 March (2007)

[16] EM Daly, M Haahr, Social network analysis for routing in disconnected delay-tolerant manets. In Proceedings of the 8th ACM international symposium on mobile ad hoc networking and computing (MobiHoc'07), pp. 32-40 (2007)

[17] Hui, P., Crowcroft, J. \&Yonek, E. BubbleRap: Socialbased forwarding in delay tolerant networks. In Proceedings of the 9th ACM international symposium on mobile Ad Hoc networking and computing (MobiHoc'08), pp. 241-250, (2008)

[18] E Bulut, B. K. Szymanski,Friendship based routing in delay tolerant mobile social networks. IEEE global telecommunications conference (GLOBECOM'10), pp. 1-5 (2010)

[19] P Hui, EYoneki, SY Chan, J Crowcroft, Distributed community detection in delay tolerant networks. In proceedings of 2nd ACM/IEEE International Workshop on Mobility in the Evolving Internet Architecture (MobiArch 2007), pp. 1-8 (2007)

[20] T Hossmann, T Spyropoulos, F Legendre, "Know thy neighbor: Towards optimal mapping of contacts to social graphs for DTN routing. In proceedings of the IEEE INFOCOM, pp. 1-9 (2010)

[21] S Batyabal, P Bhaumik, Analysing social behaviour and message dissemination in human based delay tolerant network. Wireless Networks, 21(3), 513-529 (2015)

[22] G Sun, F Xiao, L Jiang, J Xu, R Wang, A Sociability-Based Spray and Forward Scheme for Opportunistic Network. International Journal of Distributed Sensor Networks, (2013)

[23] K. Wei, X. Liang, and K. Xu, "A survey of social-aware routing protocols in delay tolerant networks: Applications, taxonomy and design-related issues," IEEE Communications Surveys Tu-torials, vol. PP, no. 99, pp. 1-23, May (2013)

[24] Y Wang, H Dang, H Wu,A survey on analytic studies of Delay-Tolerant mobile sensor networks. Wireless Communications and Mobile Computing, 7(10), 1197-1208 (2007)

[25] K. Massri, A. Vernata, A Vitaletti, Routing protocols for delay tolerant networks: a quantitative evaluation. In proceedings of the 7th ACM workshop on Performance Monitoring and Measurement of Heterogeneous Wireless and Wired Networks, pp. 107-114 (2012)

[26] S Wasserman \& K Faust, Social network analysis: Methods and applications. Cambridge: Cambridge University Press, (1994)

[27] Y Zhu, B Xu, X Shi, Y Wang, A survey of social-based routing in delay tolerant networks: positive and negative social effects, IEEECommunications Survey Tutorial, 15, (1), pp. 387-401 (2013) 
International Journal of Ad hoc, Sensor \& Ubiquitous Computing (IJASUC) Vol.7, No.1, February 2016

[28] T Karagiannis,J. L. Boudec, M.Vojnovi, Power law and exponential decay of intercontact times between mobile devices. In Proceedings of the ACM MobiCom, pp. 183-194 (2007)

[29] A Chaintreau, P Hui, C Diot, RGass, J Scott, J Crowcroft, Impact of human mobility on opportunistic forwarding algorithms. IEEE Transactions on Mobile Computing, 6(6),606-620 (2007)

[30] http://www.crawdad.cs.dartmouth.edu/index.html

[31] A Keranen, J Ott, T Karkkainen, The one simulator for dtn protocol evaluation, In proceedings of the 2nd international conference on simulation tools and techniques (Simutools 2009), pp. 1-10 (2009) 